\title{
Ashwagandha: Potential Integrative Management of Tuberculosis
}

\author{
Dr. Mukund Baburao Bandale ${ }^{1}$, Dr. Sandipkumar Ramjivan Baheti ${ }^{2}$ \\ ${ }^{1}$ Associate Professor \& H.O.D., Department of Sharira Rachana, Dr.Ved Prakash Patil Ayurveda Collegeand \\ Research Institute, Jalna, Mharashtra \\ ${ }^{2}$ Research Officer, Central Ayurved Research Institute for Respiratory Disorders, Patiala, Punjab
}

Corresponding author: Dr. Mukund Baburao Bandale, M.D. (Ayu); mukundbandale@gmail.com

Received 11 May 2020;

Accepted 29 June 2020;

Published 18 July 2020

\begin{abstract}
The World Health Organization TB (Tuberculosis) statistics for India for 2018 give an estimated incidence of 2.69 million cases.TB is being a challenge for India and worldwide developing countries pre and post antitubercular drug era. The major limitations clinicians face in management of TB is its MDR, immunosuppression, bad prognosis during course of treatment and associated side effects of anti- tubercular drugs putting patient in danger for multiple organ failure. The presence of comorbidities, nutritional deficiencies add up toll in the whole clinical presentation of tuberculosis management. There is very systematic pathophysiology and treatment ideology explained in Ayurveda for tuberculosis and related respiratory infections. There is great need to integrate Ayurveda wisdom of understanding tuberculosis and medicinal herbs in protective mode with aggressive targeted anti TB activity of modern medicine. Ashwagandha is strong Rasayana category medicinal plants with ancient references for its beneficial effect in management of tuberculosis also supported by modern day in vitro, in vivo and clinical evidences of effectiveness of Ashwagandha in management of tuberculosis could be ray of hope. There should focused research aimed at unfolding mechanisms and clinical guidelines for the use of Ashwagandha as an adjuvant in themanagement of tuberculosis.
\end{abstract}

Keywords: Tuberculosis, MDR, isoniazid, Ashwagandha

\section{Introduction}

The World Health Organization (WHO) TB (Tuberculosis) statistics for India for 2018 give an estimated incidence of 2.69 million cases. Though there are measures taken by Government towards TB. It is still prevalent in India. There are many challenges in the way of treatment for TB. The current review addresses the encounters in the clinical management of tuberculosis and emphasizes integrative way of management with Ayurveda. Furthermore the review portrays the references, in vitro, vivo and clinical research with Ashwagandha as a potential agent to be used in the clinical management. The review stresses on the more focused research need with focused research of Ashwagandha in Tuberculosis.

\section{Post DOTS scenario in India:}

Widespread implementation of the DOTS Strategy since the late 1990s has treated millions of patients with TB, and saved many lives ${ }^{[1]}$. The impact of this strategy on reducing transmission and incidence rate is not up to the mark. India could be a good example of this situation. Post National TB Control Program (RNTCP), TB incidence continues to remain high. India contributed for $25 \%$ of the 9 million global TB cases in $2013^{[2]}$. WHO assessments bring about fact that India still has missing TB cases who are either not diagnosed or not registered. TB is a key cause of death in developing countries and India, and the occurrence of severe forms of drug-resistant $\mathrm{TB}^{[3]}$.

There are several reasons to explain the persistent high $\mathrm{TB}$ incidence, TB mortality and drug-resistance in India. These includes the social conditions and co-morbidities that work as gasoline for the TB epidemic ${ }^{[4]}$. India is still believed to be holding on malnutrition badly. The association between malnutrition and $\mathrm{TB}$ in India is strong ${ }^{[5]}$. Diabetes is another important risk factor for $\mathrm{TB}$, and India is on the verge of getting epicenter of diabetes.Furthermore, tobacco smoking is strongly associated with higher risk of TB mortality ${ }^{[6]}$. The budget sanctioned to the TB program is also an important limitation of the developing countries like India. Lastly, unlike the case of HIV/AIDS, awareness around $\mathrm{TB}$ has been weak in India, contributing to limited societal participation to irradiate $\mathrm{TB}^{[7,8]}$.

\section{Challenge with Multi Drug Resistance (MDR):}

Multi-drug-resistant tuberculosis (MDR-TB) is caused by bacteria showing resistance to most powerful first-line anti-TB medications and extensively drug-resistant $\mathrm{TB}$ is called to the infections resistant to second-line medications as well ${ }^{[9]}$.

Infection of Mycobacterium tuberculosis is called as tuberculosis. This infection targets respiratory system and 
eventually spreads over vital organs in its aggressive form. In i90\% cases there are no symptoms that are very aggressive (latent tuberculosis), in $10 \%$ cases the symptoms are so profound and cases advance rapidly making prognosis deadly ${ }^{[10]}$.TB is contagious disease and air borne. Resistant strains of TB can get transferred in non-infected individual making the newly infected person a primary MDR-TB which counts for $75 \%$ cases. Another class is of Acquired MDR-TB which develops when non-resistant strain of TB is not treated properly and results in development of antibiotic resistance. Acquired MRD-TB can affect more people in community. This runs in a vicious cycle ${ }^{[11]}$.

\section{Anti-TB drugs and hepatic damage:}

Studied earlier, about 5-20\% of individuals, on anti-TB medication reported with hepatic complications ${ }^{[13]}$. Drug combinations regime in MDR-TB the hepatic damage gets more worsened. Anti-TB drugs are having known side effects as hepatic damage. Clinical symptoms of the hepatotoxicity in TB patients include, jaundice, nausea, vomiting and abdominal pain along with elevated levels of bilirubin and hepatic transaminases. The mortality rate after onset of liver disease in TB patient is $6-12 \%$ on continued drug use ${ }^{[14]}$.

\section{Need to look back at roots for help:}

In Ayurveda the tuberculosis is being explained since many thousand years together. There were treatments provided in folklore medicine before discovery of anti TB agent inmodern science. So its need to look in the Ayurvedic understanding and medicines once again to get help to manage the critical clinical scenario in management of Tuberculosis.

Nature guaranteesof plants used to cure illness of mankind. As a solid framework of traditional medical systems, herbs have made aninnumerable impact to maintain the human health. Combination of anti-TB agent together with herbs having potential as anti-mycobacterial and hepatoprotective agent could prove the key to the situation. This could lead to amalgamation of targeted therapy of anti-TB drugs with numerous health benefits of medicinal plants to manage health of patient, improve prognosis and avoid hepatotoxicity.

\section{Ayurvedic Concept of Pulmonary TB (PTB):}

PTB is suitable to be explained in Ayurvedic pathophysiological aspects as Rajayakshma.Rajayakshma prominently points outs Dhatukshaya (tissue damage or loss). This process caninitiate inflammation in the PTB pathophysiology. There are obvious metabolic deregulations happening (Dhatwagninasana), which vitiates rasa (tissue fluid), rakta (blood), mamsa (muscle), meda (adipose tissue), and sukra (generative tissue). This leads to immune-depression or ojokshaya. As per Ayurveda further metabolicdestruction leads to depletion of various dhatus (tissue) such as Ojokshaya, sukra, medadhatusto rasa dhatu can be termed as Pratilomakshaya ${ }^{[12]}$.

\section{Ashwagandha: Integrative Management of Tuberculosis:}

Withaniasomnifera (Ashawagandha) is much admired herb of the Indian Ayurvedic system of medicine as a Rasayana (tonic). It is very well researched herb for its immunomodulatory, antioxidant, anti-inflammatory, nervine tonic, revitalizing,gastro-protective and anti-stress properties. It is excellent general tonic and adaptogenic in nature ${ }^{[15]}$.

It is reported from a trusted research that, Withaniasomnifera possess activity against M. tuberculosis. Mycobacterium tuberculosis activity was tested using minimal inhibitory concentration method (MIC). Aqueous extract of W. somnifera $(0.01-1.0 \mathrm{mg} / \mathrm{mL})$ had significant effect against $\mathrm{M}$. tuberculosis.It comprehensibly provide evidence of the effective anti-tubercle activity of W. somnifera ${ }^{[16]}$.

Debnath et al. assessed the use of Ashwagandhaas an adjuvant in the management of PTB. The primary aim of study were to assess toxicity reduction of anti-TB drugs and early repair by adjunct therapy of Ayurvedic drugs improving the bioavailability of anti-TB drugsThere were 99 newly diagnosed PTB patients from both the sexes aged between 10 and 65 years completed the study. Ashwagandha $500 \mathrm{mg} 2$ caps, twice daily and Chyawanprash (as per Indian Pharmacopeia)-10 g, thrice daily for a period of 28 days was the intervention. The study reportedbetterment of symptoms, improvement of body weight, reduction inESR, IgA and IgM patterns, and significant upsurge in bioavailability of isoniazid and pyrazinamide. Bioavailability of isoniazid and pyrazinamide reported $7-10 \%$ increase after 28 days of treatment ${ }^{[17]}$.

Vyas et al. carried out a single blind controlled trial to evaluate the adjunct properties of Rasayana compound among 133 TB patients under RNTCP. The Rasayana used in this study were composed of Amalaki (EmblicaofficinalisGaertn.), Guduchi (Tinosporacordifoliawilld.), Ashwagandha (Withaniasomnifera L.), Yashtimadhu (Glycyrrhizaglabra Linn.), Pippali (Piper longum Linn) etc. as a capsule. The study duration was of 60 days. Sputum positive Category I of PTB patients or extra PTB and age group of $>13$ years were selected for the study. It was reported that the compound is helpful in relieving the symptoms of PTB in the treatment group compared to the control. The Rasayana compound containing Ashwagandha was found to decrease cough (83\%), fever $(93 \%)$, dyspnea $(71.3 \%)$, hemoptysis $(87 \%)$, and increase body weight $(7.7 \%)^{[18]}$.

As per research of Debnath et al.Aswagandhais in use for respiratory ailments. The potential activity of Ashwagandhaonposhakarasa level (nutrient supplement) agni level (metabolicappreciation) and srotas level (tissue nourishment) are

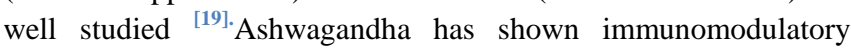
activity to boost up or restoreimmunityasresponse to defense mechanism ${ }^{[20]}$. Ashwagandha is reported to possess respiratory system specificimmunomodulatory activity leading to role of Ashwagandha in wide range of respiratory diseases ${ }^{[21]}$.Ashwagandha with building specific immunity works as an anti-stress,inotropic and antioxidant agent protecting vital organs from damage and improving symptoms and quality of life of patients.

As per study reported as a double blind placebo controlled randomized trial, with 60 freshly diagnosed sputum positive PTB patients, Ashwagandha root extract for 12 weeks with antituberculosis treatment proved significant in reducing the symptoms and relieving hepatotoxicity. Attheendof 8 weeks, $86 \%$ patients recovered on sputum test.There was significant increase in CD4 and CD8 cells in Ashwagandha treated group. There was average gain in body weight in Ashwagandha treated group with improved quality of life. There was significant difference in hepatic enzymes than placebo group with possible hepato-protection ${ }^{[22]}$.

In a clinical study undertaken as an adjuvant to anti $\mathrm{TB}$ drugs with Ashwagandha revealed that there was reduction in symptom severity cough (83\%), fever (93\%), dyspnea (71.3\%)with 
statistically significant weight gain and quality of life after study [23]

A research was intended to examine combinedtoxic effects of Isoniazid and Rifampicin (RIF), against effects of Ashwagandha and Vitamin $\mathrm{E}$ in animal models of hepatotoxicity. Ashwagandha treated group showed maximum restoration of hepatocellular integrity and improvement in biochemical parameter when compared to negative control group. This suggest potential protective effect of Ashwagandha in Anti-TB drug induced hepatotoxicity ${ }^{[24]}$.

\section{Conclusion}

The Tuberculosis is being a challenge for India and worldwide developing countries pre and post antitubercular drug era. The major limitations clinicians face in management of TB is its MDR, immunosuppression and bad prognosis during course of treatment and associated side effects of anti- tubercular drugs putting patient in danger for multiple organ failure. The presence of comorbidities, nutritional deficiencies add up toll in the whole clinical presentation of tuberculosis management. There is very systematic pathophysiology and treatment ideology explained in Ayurveda for tuberculosis and related respiratory infections. There is great need to integrate Ayurveda wisdom of understanding tuberculosis and medicinal herbs in protective mode with aggressive targeted anti TB activity of modern medicine. Ashwagandha is strong Rasayana category medicinal plants with ancient references for its beneficial effect in management of tuberculosis also supported by modern day in vitro, in vivo and clinical evidences of effectiveness of Ashwagandha in management of tuberculosis could be ray of hope. There should be more research aimed at unfolding mechanisms and clinical guidelines for the use of Ashwagandha as an adjuvant in themanagement of tuberculosis.

\section{Ethics approval and consent to participate}

Not Applicable

\section{Conflicts of Interest}

The author(s) declare(s) that there is no conflict of interest regarding the publication of this paper.

\section{Data Availability}

Not Applicable

\section{Funding Statement}

This study is an literary research work, so no any outer funding was taken.

\section{Authors' contributions}

First Author that is, Dr. Mukund Baburao Bandale did the review and search of literature of Tuberculosis and Ashwagandha and was put up contribution in writing the manuscript and editing of manuscript. Second Author, Dr. Sandipkumar Ramjivan Baheti was also involved in literature search and drafting of manuscript. Both authors review, check, read discussed multiple times and finalized the manuscript.

\section{Acknowledgments}

Nil

\section{References}

[1] WHO. Global Tuberculosis Report 2014. Geneva: World Health Organization; 2014.

[2] Dye C, Williams BG. The population dynamics and control of tuberculosis. Science 2010; 328:856-61.

[3] Sachdeva KS, Kumar A, Dewan P et al. New vision for Revised National Tuberculosis Control Programme (RNTCP): Universal access - "Reaching the un-reached". Indian J Med Res 2012; 135:690-4.

[4] Udwadia ZF. MDR, XDR, TDR tuberculosis: ominous progression. Thorax 2012; 67:286-8.

[5] Bhargava A, Sharma A, Oxlade O et al. Under nutrition and the incidence of tuberculosis in India: national and subnational estimates of the population-attributable fraction related to wundernutrition. Natl Med J India 2014; 27:128-33.

[6] International Food Policy Research Institute. Global Nutrition Report 2015: Actions and Accountability to Advance Nutrition and Sustainable Development. Washington, DC; 2015.

[7] Harries AD, Lin Y, Satyanarayana S et al. The looming epidemic of diabetes-associated tuberculosis: learning lessons from HIVassociated tuberculosis. Int $\mathbf{J}$ Tuberc Lung Dis 2011; 15:1436-44. Transactions of the Royal Society of Tropical Medicine and Hygiene 159

[8] Cheema A, Adeloye D, Sidhu S et al. Urbanization and prevalence of type 2 diabetes in Southern Asia: A systematic analysis. J Global Health 2014; 4:010404.

[9] "Diagnosis and notification of multidrug-resistant TB" (PDF). World Health Organization (WHO). Retrieved 7 December 2016.

[10] Wood, Alastair J.J.; Iseman, Michael D. (1993). "Treatment of Multidrug-Resistant Tuberculosis". New England Journal of Medicine. 329 (11): 784-91

[11] Millard, James; Ugarte-Gil, Cesar; Moore, David A. J. (26 February 2015). "Multidrug resistant tuberculosis". BMJ. 350: h882.

[12] Nathanson, Eva; Nunn, Paul; Uplekar, Mukund; Floyd, Katherine; Jaramillo, Ernesto; Lönnroth, Knut; Weil, Diana; Raviglione, Mario (9 September 2010).New England Journal of Medicine. 363 (11): 1050-1058.

[13] Charak A. In: Vidyatini Hindi Commentary, editors. CharakSamhita. Part I and II. Shastri K, Chaturvedi GN, editors. Varanasi: Chaukhamba Sanskrit Series; 1969.

[14] M.A. Arbex, M.D. Varella, H.R. Siqueira De, F.A.F. Mello De Antituberculosis drugs: drug interactions, adverse effects, and use in special situations. Part 1: firstline drugs J Bras Pneumol, 36 (2010), pp. 626-640

[15] Narendra Singh et. al., An Overview on Ashwagandha: A Rasayana (Rejuvenator) of Ayurveda, Afr J Tradit Complement Altern Med. 2011; 8(5 Suppl): 208-213.

[16] PeriyakaruppanAdaikkappanet. al., Anti-mycobacterial activity of Withaniasomnifera and Puerariatuberosa against Mycobacterium tuberculosis H37Rv, J. Acad. Indus. Res. Vol. 1(4) September 2012, 153-56.

[17] Debnath PK, Chattopadhyay J, Mitra A, Adhikari A, Alam MS, Bandopadhyay SK, et al. Adjunct therapy of Ayurvedic medicine with anti tubercular drugs on the therapeutic management of pulmonary tuberculosis. J Ayurveda Integr Med. 2012;3:141-9. 
[18] Vyas P, Chandola HM, Ghanchi F, Ranthem S. Clinical evaluation of Rasayana compound as an adjuvant in the management of tuberculosis with anti-Koch's treatment. Ayu. 2012;33: 38-43.

[19] DattaGoutam K, Debnath PK. Stress Adaptation in Ayurveda by ImmunomodulatoryRasayana in National Seminar on Rasayana Proceedings published by CCRAS, New Delhi: 2001. p. 60-75.

[20] Bhattacharya SK, Goel RK, Kaur R, Ghosal S. Antistress activity of sitoindoles VII, VIII, new sterylglycosides from Withaniasomnefera. Phytother Res 1987;1:32-3.

[21] Debnath PK, Mitra A, Hazra J, Pandit S, Biswas TK, Jana U, et al. Evidence based medicine - A clinical experience on Ayurveda Medicine in Recent Advances in Herbal Drug Research and Therapy. In: Roy A, Gulati K, editors. New Delhi: I K International Publishing House Pvt. Ltd; 2010. p. 49-73.

[22] Kumar R, Rai J, Kajal NC, Devi P. Comparative study of effect of Withaniasomnifera as an adjuvant to DOTS in patients of newly diagnosed sputum smear positive pulmonary tuberculosis, Indian J Tuberc 2017; 65(3): 246-251.

[23] Vyas P, Chandola HM, Ghanchi F, Ranthem S. Clinical evaluation of Rasayana compound as an adjuvant in the management of tuberculosis with anti-Koch's treatment. AYU 2012; 33(1): 1-6.

[24] Arundhathi S, Kumar A, Kumar R, Kumar A. Haematological and histopathological alterations due to combined toxicity of Isoniazid and Rifampicin; amelioration with Withaniasomnifera and Vitamin-E in Wistar rats. Int J Pharma Bio Sci 2015; 6(3): P222-P229. 